\title{
Photodegradation of Basic Fuchsine Dye Using Sulphur Doped Titanium Dioxide Photocatalysts
}

\author{
Lydia. C $^{1}$, Allen Gnana Raj. G \\ ${ }^{1,2}$ Department of Chemistry and Research Centre, Scott Christian College (Autonomous), \\ Nagercoil. Affiliated to Manonmaniam Sundaranar University, \\ Abishekapatti, Tirunelveli, Tamil Nadu, India.
}

\begin{abstract}
Photocatalyst preparation using titanium dioxide and thiourea was investigated broadly for the basic fuchsine dye degradation in double distilled water. Thus the sulphur doped titanium dioxide photocatalysts prepared in the ratio of $1: 1,1: 2$ were analyzed and used in the photodegradation process. The photodegradation was conducted in artificial light with different concentrations. The photocatalysts were analyzed with various techniques. The doped samples synthesized by steaming methods and by the use of furnace revealed appreciable degradation values in the photodegradation from the dye's initial concentration. Ultraviolet visible spectrum reveals the absorption shift of the dye to visible light range. Various parameters like concentration of dopants, catalytic concentration added to the dye, $\mathrm{pH}$ changes were observed during the process. Scanning electron microscopic images, X-Ray diffraction studies of the catalysts, Energy dispersive $\mathrm{X}$ ray analysis and Infrared spectrums were taken for the samples.

Keywords: titanium dioxide, thiourea, photocatalyst, photodegradation, basic fuchsine dye
\end{abstract}

\section{Introduction}

Dyes are compounds of resistance in industries and are in the waste waters which are from them. The effluents from paper, leather, and ceramic industries have dye components which affect the environment [1]. The photocatalytic activity of titanium dioxide has been extensively studied and it has a wide band gap and the absorption range is in the UV region and studies have been made to increase the absorption range to the visible light region of the spectrum [2]. In this paper, work has been done to increase the absorption range by adding non metal as dopant using thiourea as starting material to bind with titanium dioxide.

\section{Review of Literature}

Young Seak Lee et al., has investigated the effect of carbon, nitrogen, boron and fluorine doping on titanium dioxide with tetraethylammonium tetrafluoroborate used in solar photodegradation of dyes like Acridine orange and Methylene blue which has been analyzed by XRD, XPS studies. The band gap was reduced successfully in this [3].

\section{Materials and Methods}

Basic Fuchsine dye from Sigma Aldrich, double distilled water, Titanium dioxide from Spectrum Chemicals, Thiourea from Spectrum Chemicals, Hydrochloric acid, Steam bath and Muffle furnace were required for the experiment.

\section{Experimental Procedure}

Thiourea was taken in grams, stirred and dissolved in double distilled water, after it was dissolved; measured amount of titanium dioxide was added to the mixture [4]. The beaker with the contents were kept in the steam bath and evaporated. The powdered sample was scrapped off and transferred to the crucible and kept in muffle furnace for 4 hours. The ratio of thiourea and titanium dioxide taken was $1: 1$, $2: 1$ at $400^{\circ} \mathrm{C}, 500^{\circ} \mathrm{C}, 600^{\circ} \mathrm{C}$ and the photocatalysts were prepared. The photodegradation of the dissolved basic fuchsine dye was conducted by adding the certain catalytic amount and the samples were taken out at a time interval of 30 minutes and checked for absorbance value using Ultraviolet spectroscopy

[5]. 


\section{Results and Discussion pH Determination}

\begin{tabular}{|l|l|}
\hline Before dye degradation & 6.4 \\
\hline After dye degradation & $6.4-8.9$ \\
\hline
\end{tabular}

\section{Tables and Figures}

Table: 1 Dye samples in dark

\begin{tabular}{|l|l|l|}
\hline time (hours) & wavelength(nm) & absorbance(a.u) \\
\hline $0-3$ & $537.2\left(\mathrm{I}_{0}\right)$ & 0.956 \\
\hline
\end{tabular}

Table: 2 Dye samples in light alone

\begin{tabular}{|l|l|l|}
\hline time & Wavelength & absorbance \\
\hline 0 & 537.2 & 0.956 \\
\hline 0.5 & & 0.935 \\
\hline 1 & & 0.920 \\
\hline 1.5 & & 0.916 \\
\hline 2 & & 0.880 \\
\hline 2.5 & & 0.870 \\
\hline 3 & & 0.800 \\
\hline
\end{tabular}

pc- photocatalyst

Table: 3 Dye samples in light with $0.01 \mathrm{~g}, 1: 1,400^{\circ} \mathrm{C} \mathrm{S}, \mathrm{N}-$ $\mathrm{TiO}_{2} \mathrm{pc}$

\begin{tabular}{|l|l|l|}
\hline time & wavelength & absorbance \\
\hline 0 & 537.2 & 0.956 \\
\hline 0.5 & & 0.604 \\
\hline 1 & & 0.594 \\
\hline 1.5 & & 0.533 \\
\hline 2 & & 0.513 \\
\hline 2.5 & & 0.479 \\
\hline 3 & & 0.368 \\
\hline
\end{tabular}

Table: 4 Dye samples in light with $0.01 \mathrm{~g}, 1: 2,400^{\circ} \mathrm{C} \mathrm{S,N}-$ $\mathrm{TiO}_{2} \mathrm{pc}$

\begin{tabular}{|l|l|l|}
\hline time & wavelength & absorbance \\
\hline 0 & 537.2 & 0.956 \\
\hline 0.5 & & 0.606 \\
\hline 1 & & 0.605 \\
\hline 1.5 & & 0.519 \\
\hline 2 & & 0.501 \\
\hline 2.5 & & 0.491 \\
\hline 3 & & 0.47 \\
\hline
\end{tabular}

Table: 5 Dye samples in light with $0.01 \mathrm{~g}, 1: 1,500^{\circ} \mathrm{C} \mathrm{S,N-}$

\begin{tabular}{|l|l|l|}
\hline \multicolumn{2}{|c|}{$\mathrm{TiO}_{2} \mathrm{pc}$} & absorbance \\
\hline time & wavelength & 0.956 \\
\hline 0.5 & 537.2 & 0.494 \\
\hline 1 & & 0.397 \\
\hline 1.5 & & 0.382 \\
\hline 2 & & 0.322 \\
\hline 2.5 & & 0.301 \\
\hline 3 & & 0.269 \\
\hline
\end{tabular}

Table: 6 Dye samples in light with $0.01 \mathrm{~g}, 1: 2,500^{\circ} \mathrm{C} \mathrm{S}, \mathrm{N}-$

\begin{tabular}{|l|l|l|}
\hline \multicolumn{3}{|c|}{$\mathrm{TiO}_{2} \mathrm{pc}$} \\
\hline time & wavelength & absorbance \\
\hline 0 & 537.2 & 0.956 \\
\hline 0.5 & & 0.45 \\
\hline 1 & & 0.432 \\
\hline 1.5 & & 0.406 \\
\hline 2 & & 0.392 \\
\hline 2.5 & & 0.391 \\
\hline 3 & & 0.317 \\
\hline
\end{tabular}


Table: 7 Dye samples in light with $0.01 \mathrm{~g}, 1: 1,600^{\circ} \mathrm{C} \mathrm{S}, \mathrm{N}$ $\mathrm{TiO}_{2} \mathrm{pc}$

\begin{tabular}{|l|l|l|}
\hline time & wavelength & absorbance \\
\hline 0 & 537.2 & 0.956 \\
\hline 0.5 & & 0.558 \\
\hline 1 & & 0.512 \\
\hline 1.5 & & 0.502 \\
\hline 2 & & 0.484 \\
\hline 2.5 & & 0.445 \\
\hline 3 & & 0.436 \\
\hline
\end{tabular}

Table: 8 Dye samples in light with $0.01 \mathrm{~g}, 1: 2,600^{\circ} \mathrm{C} \mathrm{S}, \mathrm{N}-$ $\mathrm{TiO}_{2} \mathrm{pc}$

\begin{tabular}{|l|l|l|}
\hline time & wavelength & absorbance \\
\hline 0 & 537.2 & 0.956 \\
\hline 0.5 & & 0.569 \\
\hline 1 & & 0.544 \\
\hline 1.5 & & 0.524 \\
\hline 2 & & 0.488 \\
\hline 2.5 & & 0.450 \\
\hline 3 & & 0.438 \\
\hline
\end{tabular}

Table: 9 Dye samples in light with $0.02 \mathrm{~g}, 1: 1,400^{\circ} \mathrm{C} \mathrm{S}, \mathrm{N}$ -

$$
\mathrm{TiO}_{2} \mathrm{pc}
$$

\begin{tabular}{|l|l|l|}
\hline time & wavelength & absorbance \\
\hline 0 & 537.2 & 0.956 \\
\hline 0.5 & & 0.623 \\
\hline 1 & & 0.597 \\
\hline 1.5 & & 0.538 \\
\hline 2 & & 0.515 \\
\hline 2.5 & & 0.406 \\
\hline 3 & & 0.404 \\
\hline
\end{tabular}

Table: 10 Dye samples in light with $0.02 \mathrm{~g}, 1: 2,400^{\circ} \mathrm{C} \mathrm{S}, \mathrm{N}-$ $\mathrm{TiO}_{2} \mathrm{pc}$

\begin{tabular}{|l|l|l|}
\hline time & wavelength & absorbance \\
\hline 0 & 537.2 & 0.956 \\
\hline 0.5 & & 0.546 \\
\hline 1 & & 0.541 \\
\hline 1.5 & & 0.516 \\
\hline 2 & & 0.491 \\
\hline 2.5 & & 0.455 \\
\hline 3 & & 0.428 \\
\hline
\end{tabular}

Table: 11 Dye samples in light with $0.02 \mathrm{~g}, 1: 1,500^{\circ} \mathrm{C} \mathrm{S,N-}$ $\mathrm{TiO}_{2} \mathrm{pc}$

\begin{tabular}{|l|l|l|}
\hline time & wavelength & absorbance \\
\hline 0 & 537.2 & 0.956 \\
\hline 0.5 & & 0.549 \\
\hline 1 & & 0.497 \\
\hline 1.5 & & 0.475 \\
\hline 2 & & 0.468 \\
\hline 2.5 & & 0.441 \\
\hline 3 & & 0.355 \\
\hline
\end{tabular}

Table: 12 Dye samples in light with $0.02 \mathrm{~g}, 1: 2,500^{\circ} \mathrm{C} \mathrm{S}, \mathrm{N}-$ $\mathrm{TiO}_{2} \mathrm{pc}$

\begin{tabular}{|l|l|l|}
\hline time & wavelength & absorbance \\
\hline 0 & 537.2 & 0.956 \\
\hline 0.5 & & 0.693 \\
\hline 1 & & 0.594 \\
\hline 1.5 & & 0.586 \\
\hline 2 & & 0.47 \\
\hline 2.5 & & 0.46 \\
\hline 3 & & 0.459 \\
\hline
\end{tabular}


Table: 13 Dye samples in light with $0.02 \mathrm{~g}, 1: 1,600^{\circ} \mathrm{C} \mathrm{S}, \mathrm{N}-$ $\mathrm{TiO}_{2} \mathrm{pc}$

\begin{tabular}{|l|l|l|}
\hline time & wavelength & absorbance \\
\hline 0 & 537.2 & 0.956 \\
\hline 0.5 & & 0.723 \\
\hline 1 & & 0.664 \\
\hline 1.5 & & 0.629 \\
\hline 2 & & 0.61 \\
\hline 2.5 & & 0.606 \\
\hline 3 & & 0.602 \\
\hline
\end{tabular}

Table: 14 Dye samples in light with $0.02 \mathrm{~g}, 1: 2,600^{\circ} \mathrm{C} \mathrm{S}, \mathrm{N}-$ $\mathrm{TiO}_{2} \mathrm{pc}$

\begin{tabular}{|l|l|l|}
\hline time & wavelength & absorbance \\
\hline 0 & 537.2 & 0.956 \\
\hline 0.5 & & 0.754 \\
\hline 1 & & 0.676 \\
\hline 1.5 & & 0.67 \\
\hline 2 & & 0.652 \\
\hline 2.5 & & 0.634 \\
\hline 3 & & 0.629 \\
\hline
\end{tabular}

Table: 15 Dye samples in light with $0.03 \mathrm{~g}, 1: 1,400^{\circ} \mathrm{C} \mathrm{S,N}$ $\mathrm{TiO}_{2} \mathrm{pc}$

\begin{tabular}{|l|l|l|}
\hline time & wavelength & absorbance \\
\hline 0 & 537.2 & 0.956 \\
\hline 0.5 & & 0.859 \\
\hline 1 & & 0.853 \\
\hline 1.5 & & 0.851 \\
\hline 2 & & 0.840 \\
\hline 2.5 & & 0.757 \\
\hline 3 & & 0.751 \\
\hline
\end{tabular}

Table: 16 Dye samples in light with $0.03 \mathrm{~g}, 1: 2,400^{\circ} \mathrm{C} \mathrm{S}, \mathrm{N}-$ $\mathrm{TiO}_{2} \mathrm{pc}$

\begin{tabular}{|l|l|l|}
\hline time & wavelength & absorbance \\
\hline 0 & 537.2 & 0.956 \\
\hline 0.5 & & 0.894 \\
\hline 1 & & 0.878 \\
\hline 1.5 & & 0.863 \\
\hline 2 & & 0.856 \\
\hline 2.5 & & 0.849 \\
\hline 3 & & 0.796 \\
\hline
\end{tabular}

Table: 17 Dye samples in light with $0.03 \mathrm{~g}, 1: 1,500^{\circ} \mathrm{C} \mathrm{S}, \mathrm{N}-$ $\mathrm{TiO}_{2} \mathrm{pc}$

\begin{tabular}{|l|l|l|}
\hline time & wavelength & absorbance \\
\hline 0 & 537.2 & 0.956 \\
\hline 0.5 & & 0.803 \\
\hline 1 & & 0.765 \\
\hline 1.5 & & 0.732 \\
\hline 2 & & 0.673 \\
\hline 2.5 & & 0.617 \\
\hline 3 & & 0.599 \\
\hline
\end{tabular}

Table: 18 Dye samples in light with $0.03 \mathrm{~g}, 1: 2,500^{\circ} \mathrm{C} \mathrm{S}, \mathrm{N}-$ $\mathrm{TiO}_{2} \mathrm{pc}$

\begin{tabular}{|l|l|l|}
\hline time & wavelength & absorbance \\
\hline 0 & 537.2 & 0.956 \\
\hline 0.5 & & 0.791 \\
\hline 1 & & 0.779 \\
\hline 1.5 & & 0.755 \\
\hline 2 & & 0.736 \\
\hline 2.5 & & 0.730 \\
\hline 3 & & 0.700 \\
\hline
\end{tabular}


Table: 19 Dye samples in light with $0.03 \mathrm{~g}, 1: 1,600^{\circ} \mathrm{C} \mathrm{S}, \mathrm{N}-$ $\mathrm{TiO}_{2} \mathrm{pc}$

\begin{tabular}{|l|l|l|}
\hline time & wavelength & absorbance \\
\hline 0 & 537.2 & 0.956 \\
\hline 0.5 & & 0.931 \\
\hline 1 & & 0.818 \\
\hline 1.5 & & 0.799 \\
\hline 2 & & 0.762 \\
\hline 2.5 & & 0.750 \\
\hline 3 & & 0.507 \\
\hline
\end{tabular}

Table: 20 Dye samples in light with $0.03 \mathrm{~g}, 1: 2,600^{\circ} \mathrm{C} \mathrm{S}, \mathrm{N}$ $\mathrm{TiO}_{2} \mathrm{pc}$

\begin{tabular}{|l|l|l|}
\hline time & wavelength & absorbance \\
\hline 0 & 537.2 & 0.956 \\
\hline 0.5 & & 0.896 \\
\hline 1 & & 0.869 \\
\hline 1.5 & & 0.862 \\
\hline 2 & & 0.829 \\
\hline 2.5 & & 0.814 \\
\hline 3 & & 0.546 \\
\hline
\end{tabular}

Ultraviolet Spectroscopy of the photodegraded dye samples

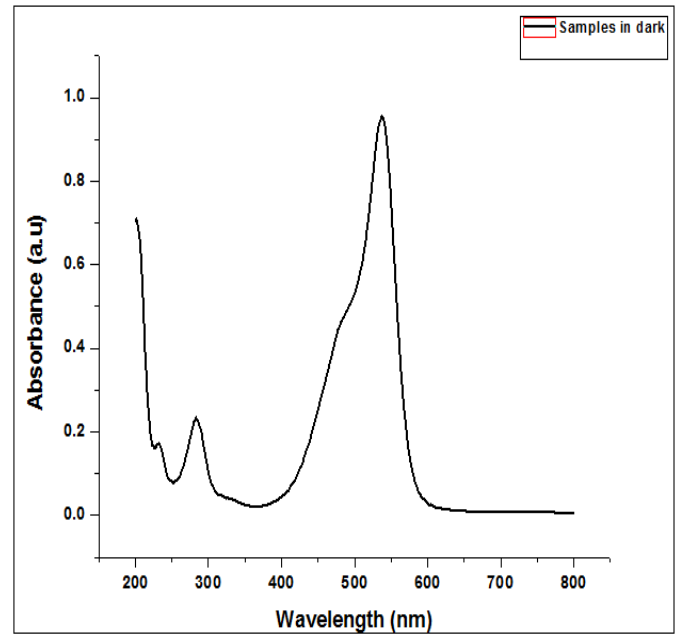

Fig 1: Samples in dark containing 0.956a.u absorbance for 3 hours

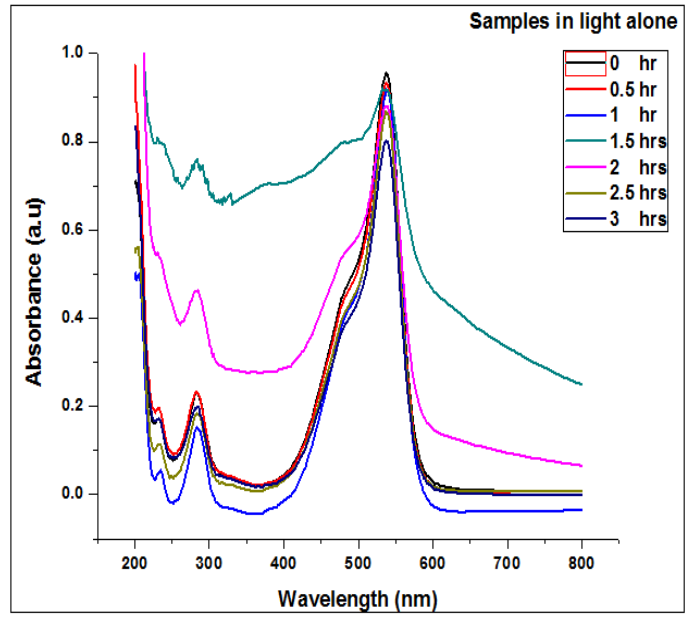

Fig 2: Samples in light alone

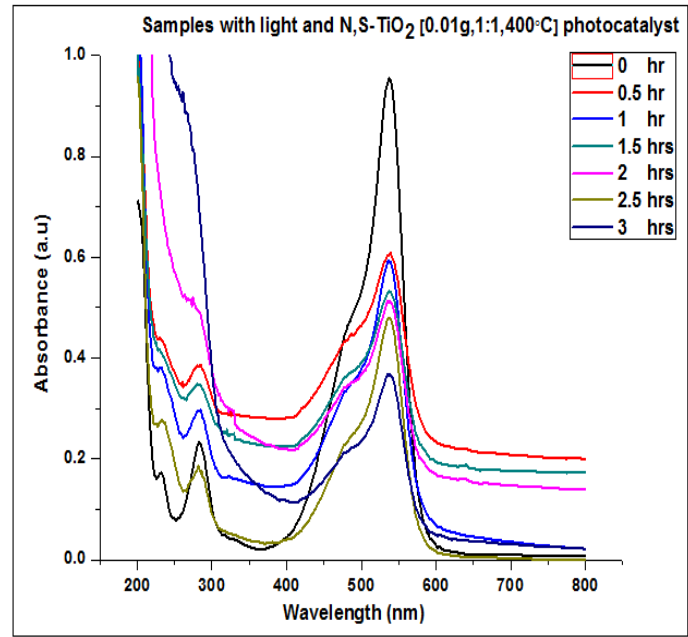

Fig 3: Samples in light with $0.01 \mathrm{~g}, 1: 1,400^{0} \mathrm{C} \mathrm{pc}$ 


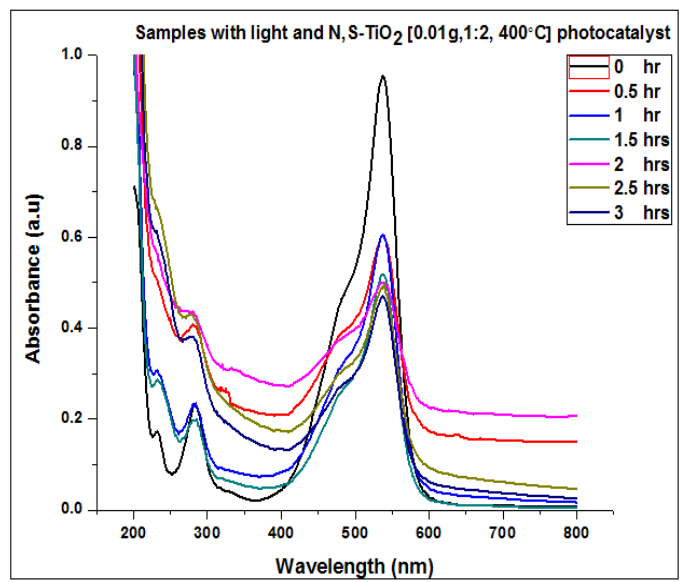

Fig 4: Samples in light with $0.01 \mathrm{~g}, 1: 2,400^{\circ} \mathrm{C} \mathrm{pc}$

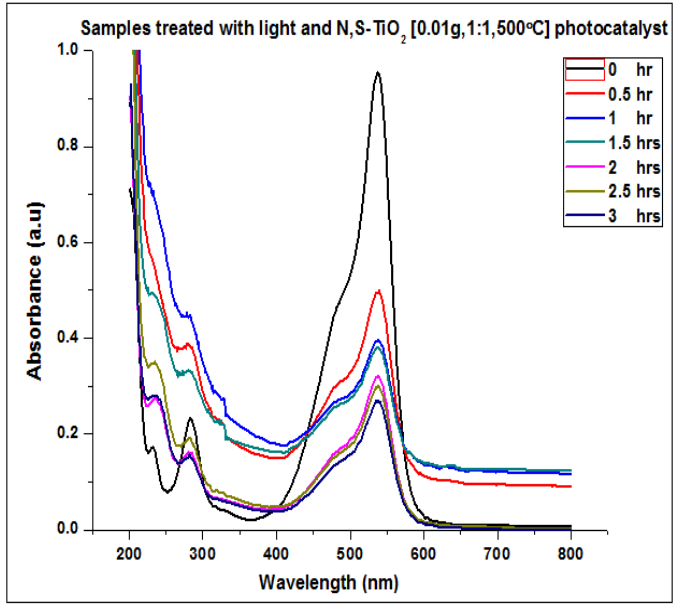

Fig 5: Samples in light with $0.01 \mathrm{~g}, 1: 1,500^{\circ} \mathrm{C} \mathrm{pc}$

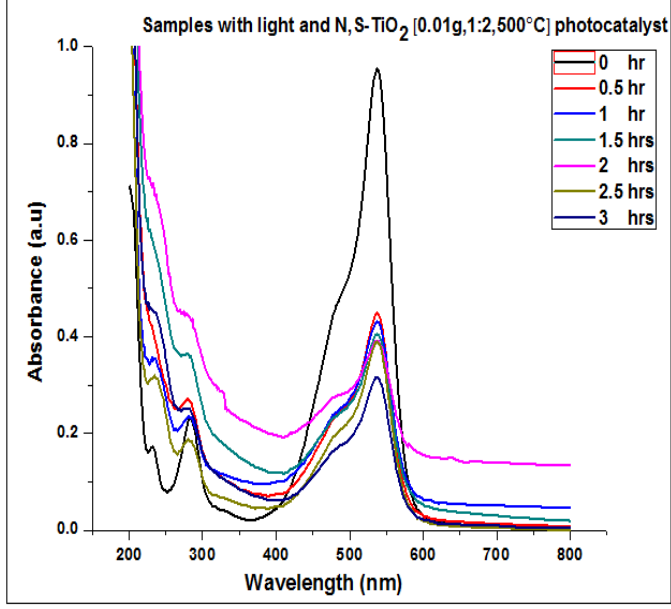

Fig 6: Samples in light with $0.01 \mathrm{~g}, 1: 2,500^{0} \mathrm{C} \mathrm{pc}$

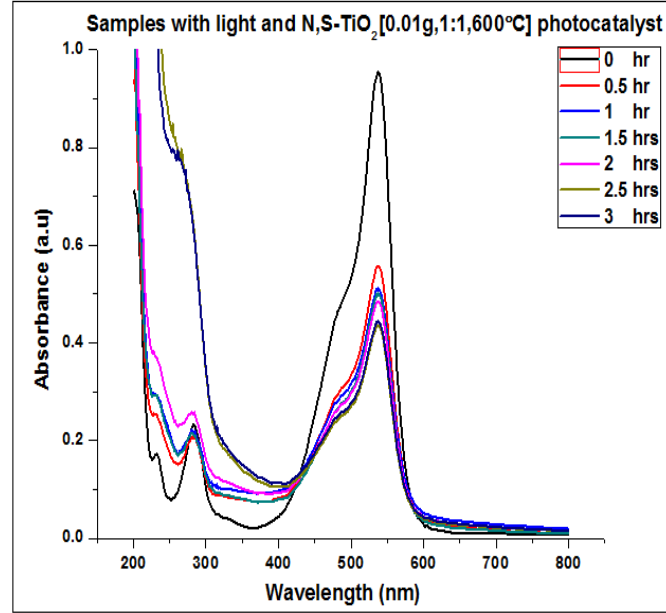

Fig 7: Samples in light with $0.01 \mathrm{~g}, 1: 1,600^{\circ} \mathrm{C} \mathrm{pc}$

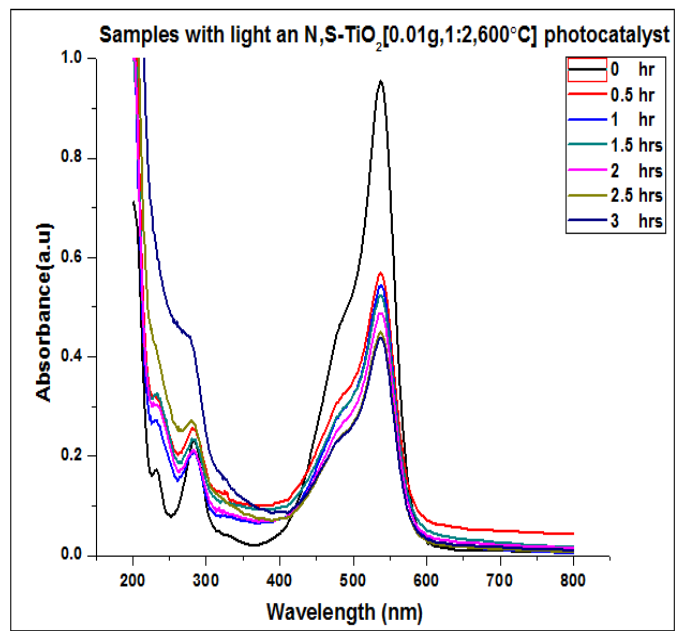

Fig 8: Samples in light with $0.01 \mathrm{~g}, 1: 2,600^{\circ} \mathrm{C} \mathrm{pc}$

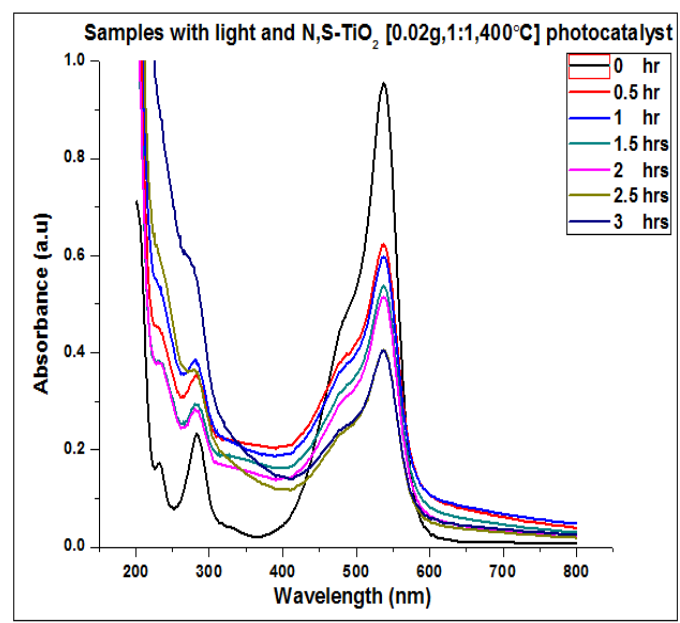

Fig 9: Samples in light with $0.02 \mathrm{~g}, 1: 1,400^{\circ} \mathrm{C} \mathrm{pc}$ 


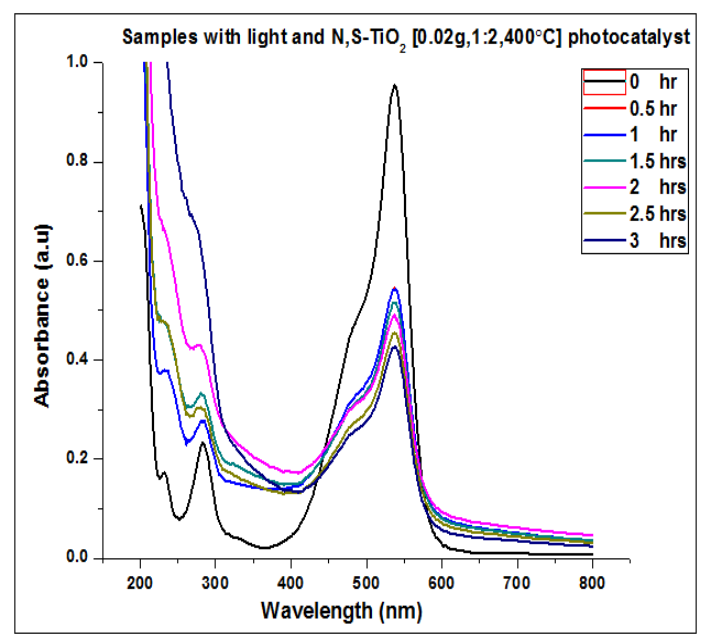

Fig 10: Samples in light with $0.02 \mathrm{~g}, 1: 2,400^{\circ} \mathrm{C}$ pc

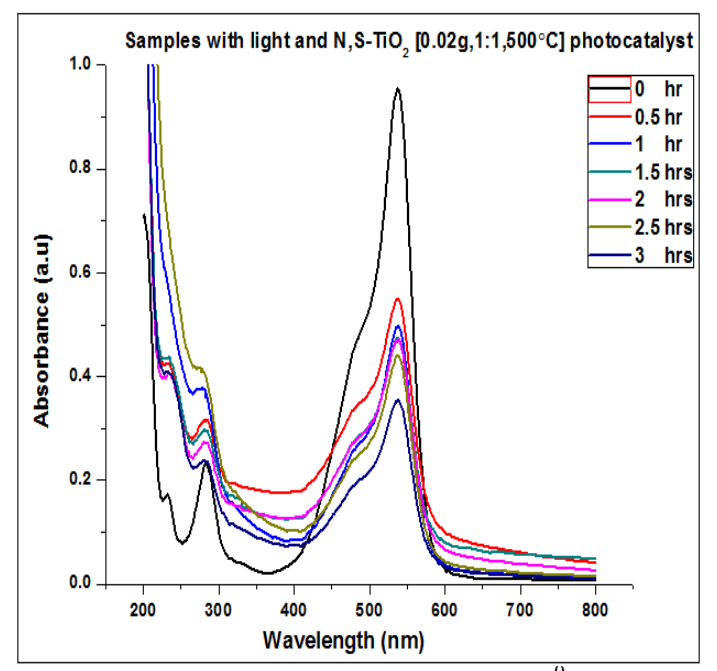

Fig 11: Samples in light with $0.02 \mathrm{~g}, 1: 1,500^{0} \mathrm{C} \mathrm{pc}$

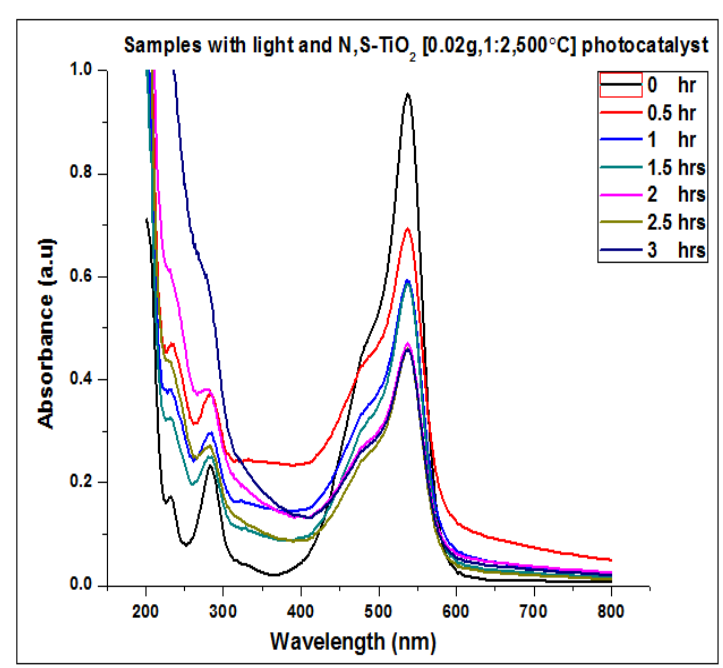

Fig 12: Samples in light with $0.02 \mathrm{~g}, 1: 2,500^{0} \mathrm{C} \mathrm{pc}$

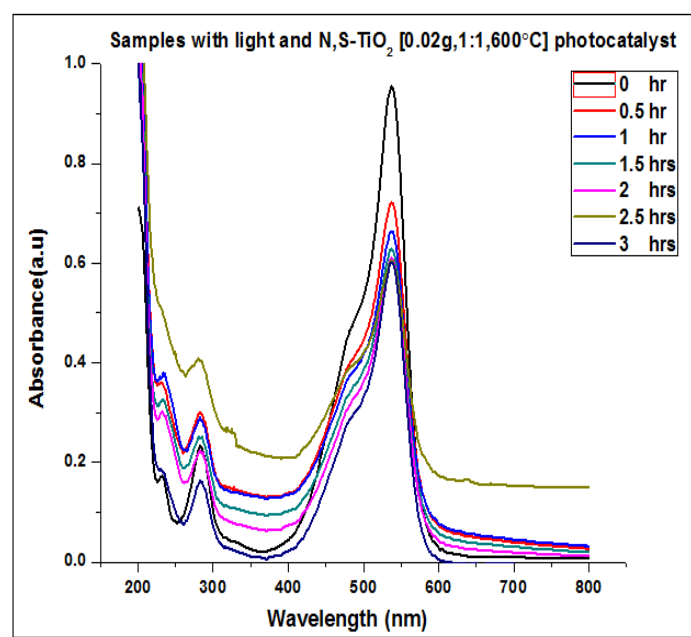

Fig 13: Samples in light with $0.02 \mathrm{~g}, 1: 1,600^{\circ} \mathrm{C} \mathrm{pc}$

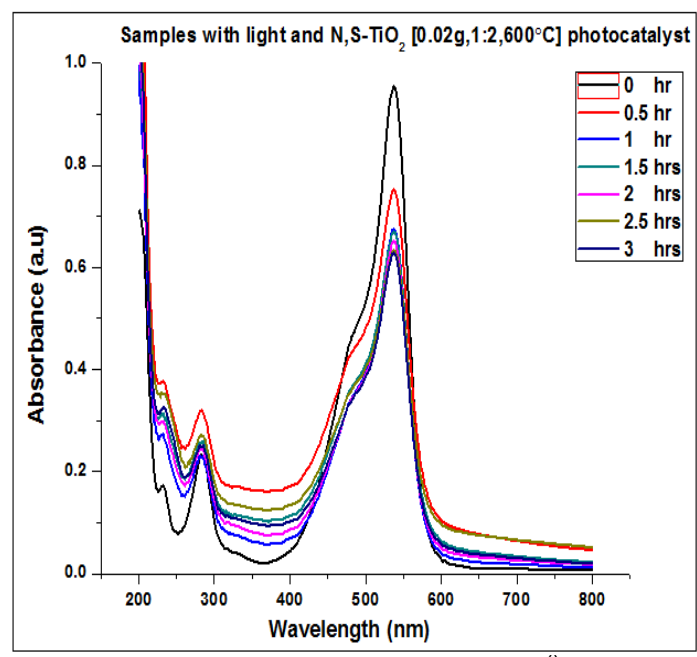

Fig 14: Samples in light with $0.02 \mathrm{~g}, 1: 2,600^{\circ} \mathrm{C} \mathrm{pc}$

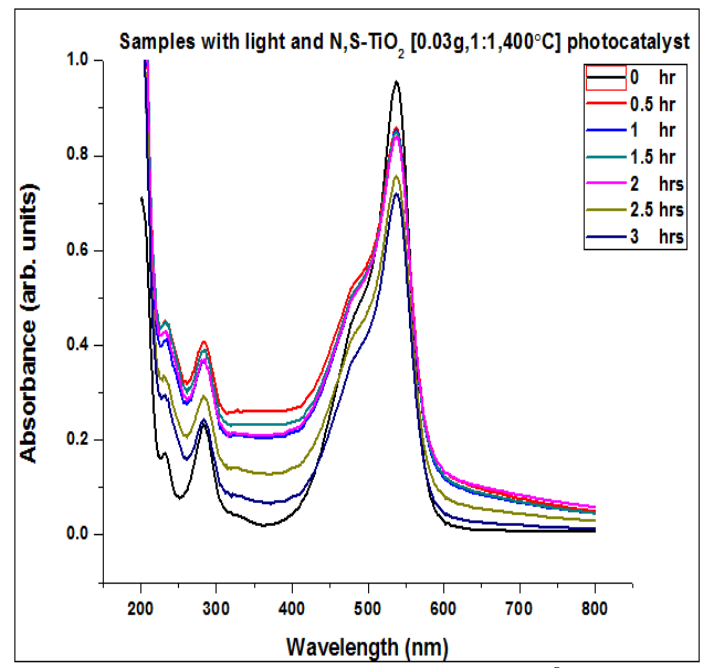

Fig 15: Samples in light with $0.03 \mathrm{~g}, 1: 1,400^{\circ} \mathrm{C} \mathrm{pc}$ 


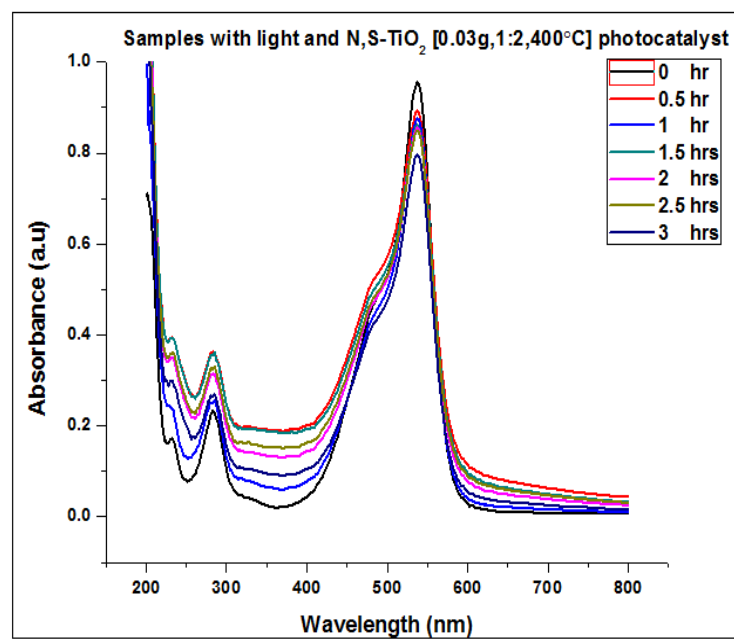

Fig 16: Samples in light with $0.03 \mathrm{~g}, 1: 2,400^{0} \mathrm{C} \mathrm{pc}$

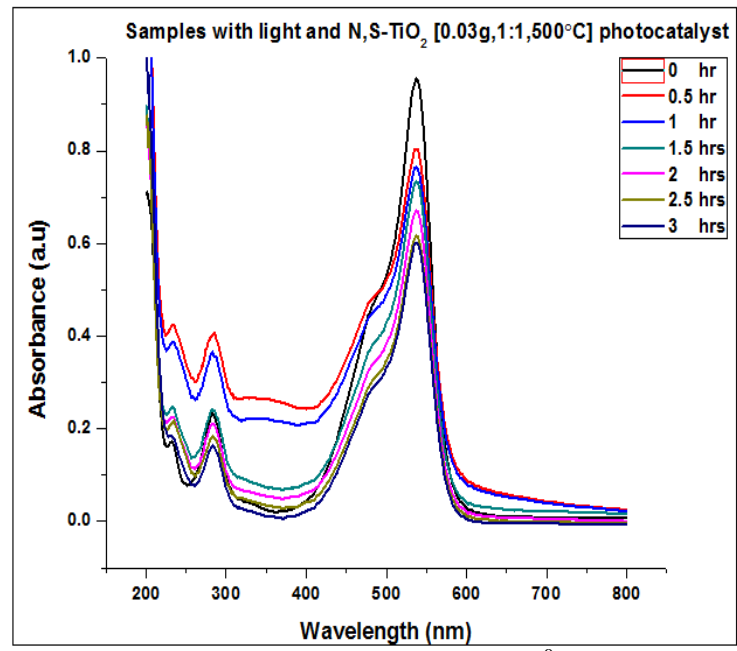

Fig 17: Samples in light with $0.03 \mathrm{~g}, 1: 1,500^{\circ} \mathrm{C} \mathrm{pc}$

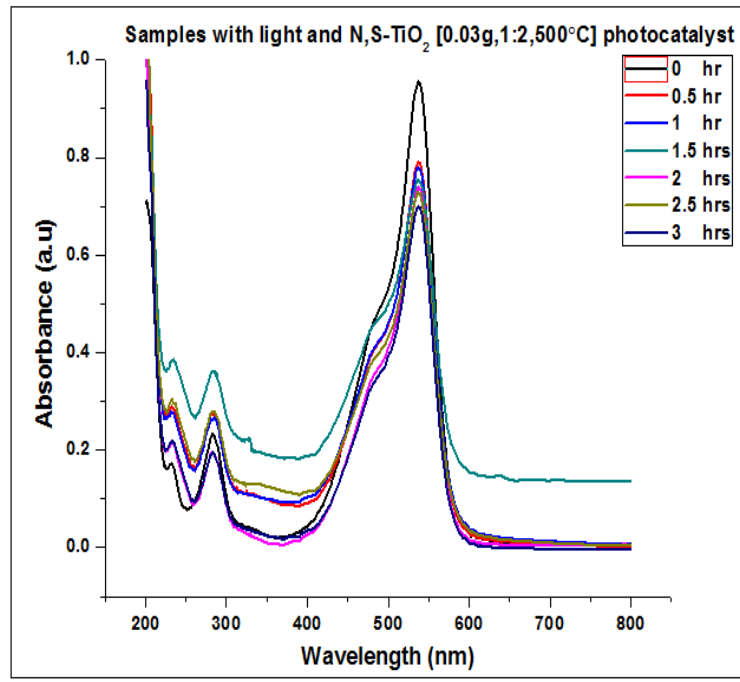

Fig 18: Samples in light with $0.03 \mathrm{~g}, 1: 2,500^{\circ} \mathrm{C} \mathrm{pc}$

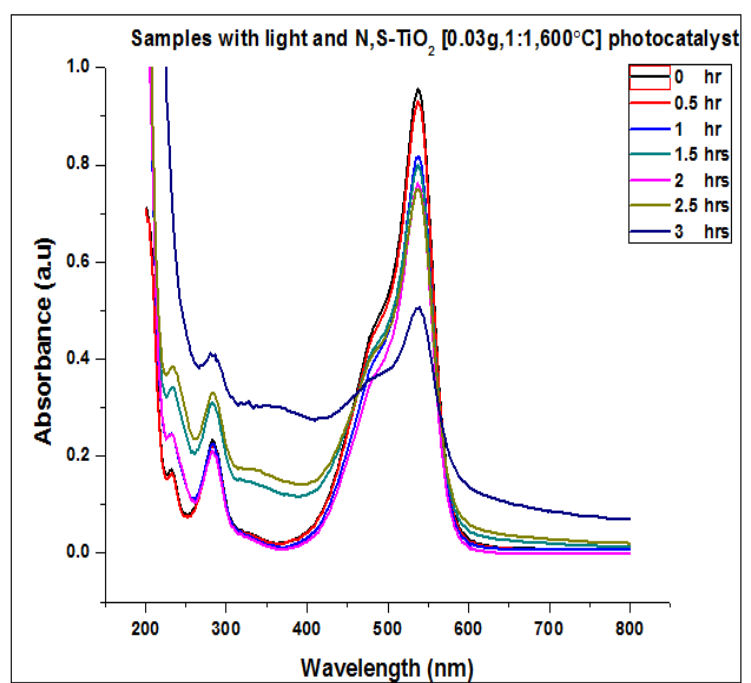

Fig 19: Samples in light with $0.03 \mathrm{~g}, 1: 1,600^{\circ} \mathrm{C} \mathrm{pc}$

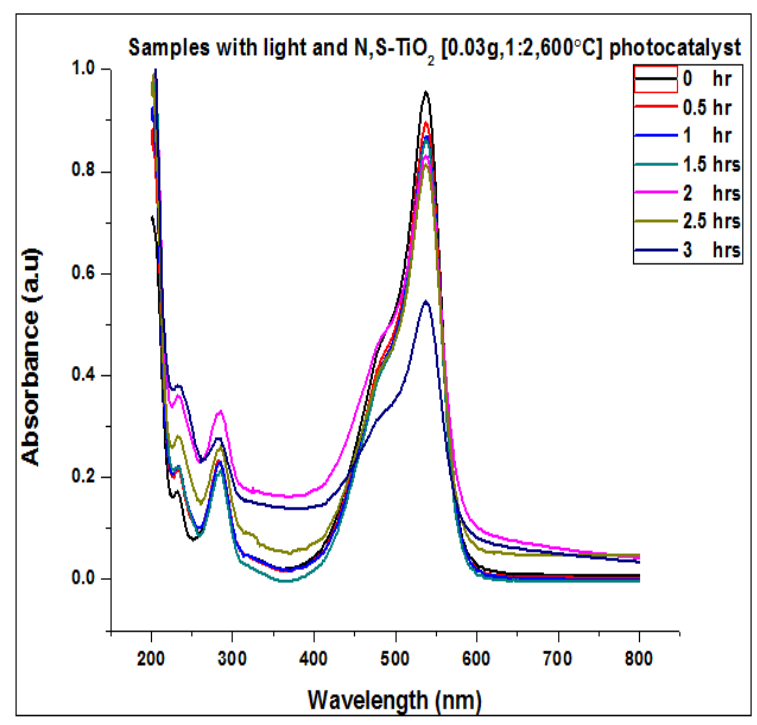

Fig 20: Samples in light with $0.03 \mathrm{~g}, 1: 2,600^{\circ} \mathrm{C} \mathrm{pc}$

Energy Dispersive X-Ray Analysis of sulphur doped titanium dioxide pc

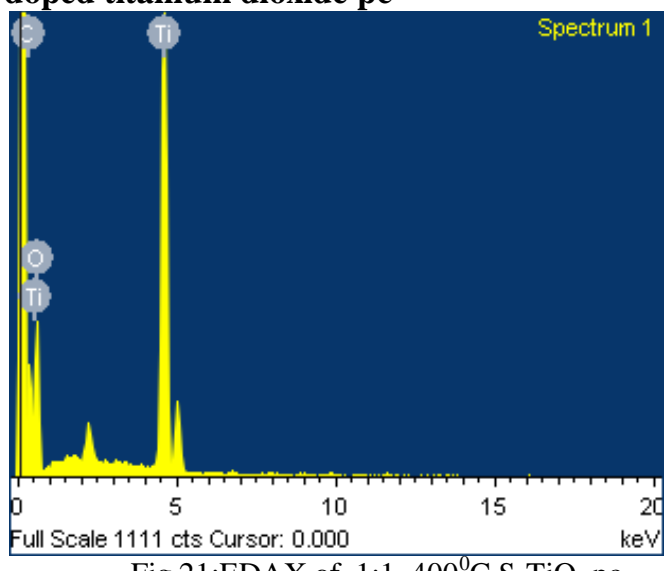

Fig 21:EDAX of $1: 1,400^{\circ} \mathrm{C} \mathrm{S}-\mathrm{TiO}_{2} \mathrm{pc}$ 


\begin{tabular}{|l|l|l|}
\hline Element & $\begin{array}{l}\text { Intensity } \\
\text { Correlation }\end{array}$ & Atomic \% \\
\hline $\mathrm{C}$ & 0.8653 & 25.02 \\
\hline $\mathrm{O}$ & 0.3672 & 59.61 \\
\hline $\mathrm{Ti}$ & 0.8714 & 15.37 \\
\hline
\end{tabular}

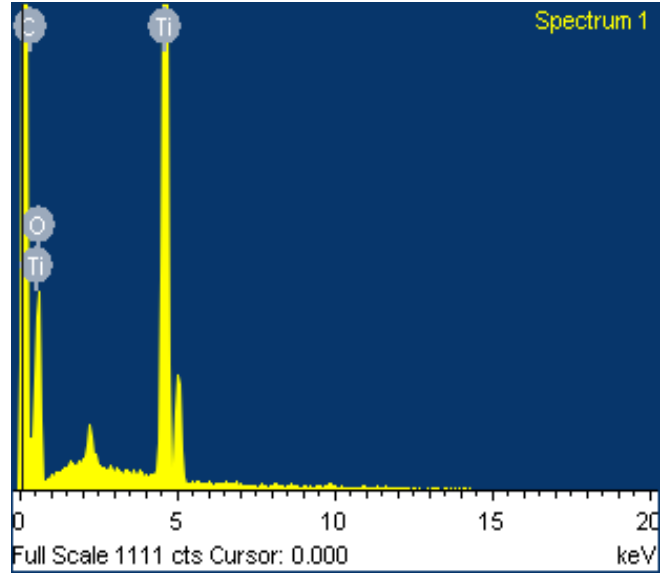

Fig 22:EDAX of $1: 2,400^{\circ} \mathrm{C} \mathrm{S}-\mathrm{TiO}_{2} \mathrm{pc}$

\begin{tabular}{|l|l|l|}
\hline Element & $\begin{array}{l}\text { Intensity } \\
\text { Correlation }\end{array}$ & Atomic \% \\
\hline $\mathrm{C}$ & 0.8056 & 10.12 \\
\hline $\mathrm{O}$ & 0.3458 & 68.50 \\
\hline $\mathrm{Ti}$ & 0.8899 & 21.38 \\
\hline
\end{tabular}

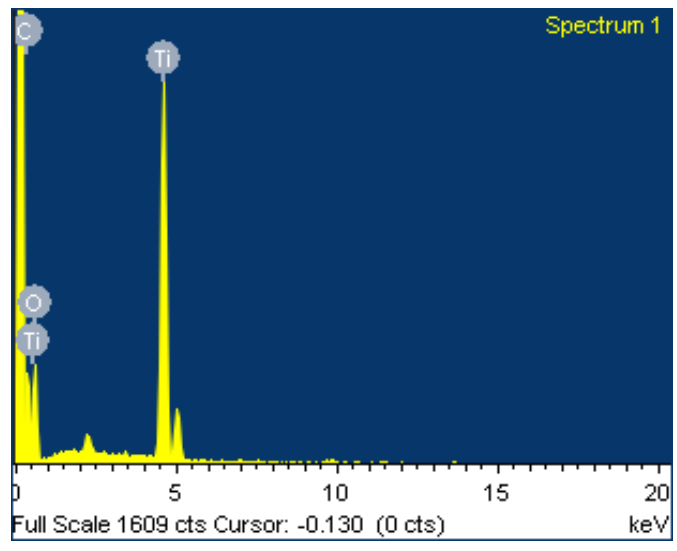

Fig 23:EDAX of $1: 1,500^{\circ} \mathrm{C} \mathrm{S}-\mathrm{TiO}_{2} \mathrm{pc}$

\begin{tabular}{|l|l|l|}
\hline Element & $\begin{array}{l}\text { Intensity } \\
\text { Correlation }\end{array}$ & Atomic \% \\
\hline $\mathrm{C}$ & 0.8746 & 28.45 \\
\hline $\mathrm{O}$ & 0.3442 & 55.23 \\
\hline $\mathrm{Ti}$ & 0.8744 & 16.32 \\
\hline
\end{tabular}

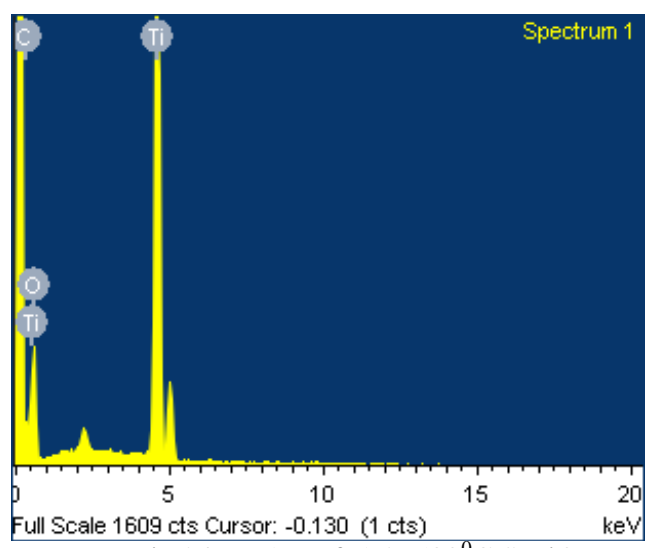

Fig 24:EDAX of $1: 2,500^{\circ} \mathrm{C} \mathrm{S}-\mathrm{TiO}_{2} \mathrm{pc}$

\begin{tabular}{|l|l|l|}
\hline Element & $\begin{array}{l}\text { Intensity } \\
\text { Correlation }\end{array}$ & Atomic \% \\
\hline $\mathrm{C}$ & 0.8048 & 10.93 \\
\hline $\mathrm{O}$ & 0.3264 & 65.77 \\
\hline $\mathrm{Ti}$ & 0.8950 & 23.30 \\
\hline
\end{tabular}

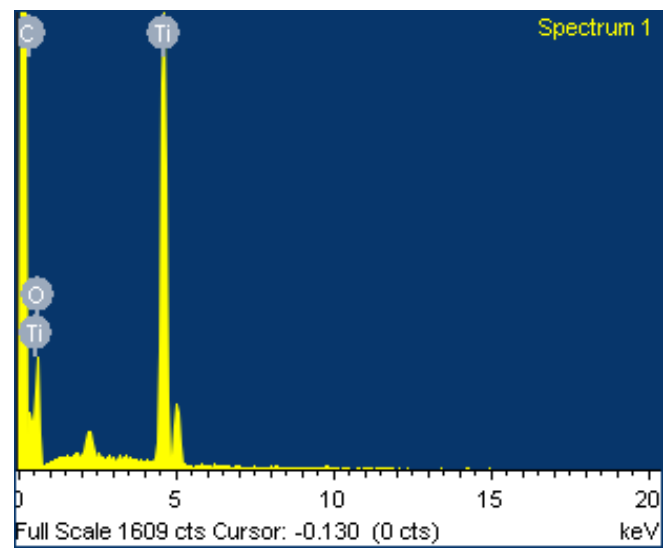

Fig 25:EDAX of $1: 1,600^{\circ} \mathrm{C} \mathrm{S}-\mathrm{TiO}_{2} \mathrm{pc}$

\begin{tabular}{|l|l|l|}
\hline Element & $\begin{array}{l}\text { Intensity } \\
\text { Correlation }\end{array}$ & Atomic \% \\
\hline $\mathrm{C}$ & 0.8343 & 19.22 \\
\hline $\mathrm{O}$ & 0.3357 & 60.97 \\
\hline $\mathrm{Ti}$ & 0.8853 & 19.81 \\
\hline
\end{tabular}

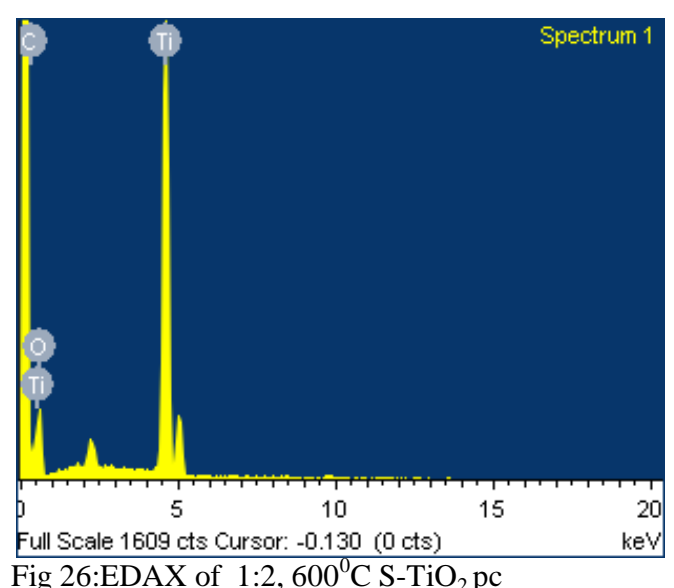

Fig 26:EDAX of $1: 2,600^{\circ} \mathrm{C} \mathrm{S}-\mathrm{TiO}_{2} \mathrm{pc}$ 


\begin{tabular}{|l|l|l|}
\hline Element & $\begin{array}{l}\text { Intensity } \\
\text { Correlation }\end{array}$ & Atomic \% \\
\hline $\mathrm{C}$ & 0.8024 & 11.96 \\
\hline $\mathrm{O}$ & 0.2999 & 61.39 \\
\hline $\mathrm{Ti}$ & 0.9036 & 26.65 \\
\hline
\end{tabular}

\section{Scanning Electron Microscopic Images of sulphur} doped titanium dioxide pc

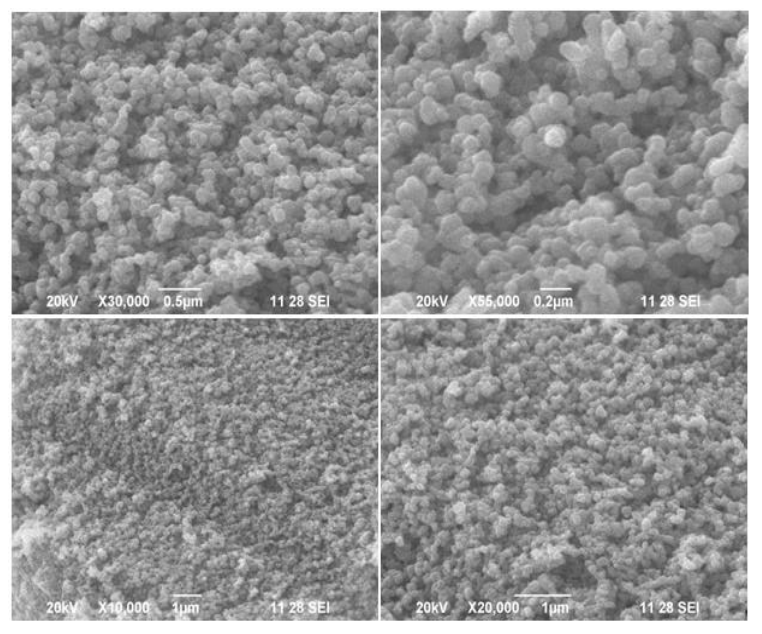

Fig 27: SEM of $1: 1,400^{\circ} \mathrm{C} \mathrm{S}-\mathrm{TiO}_{2} \mathrm{pc}$

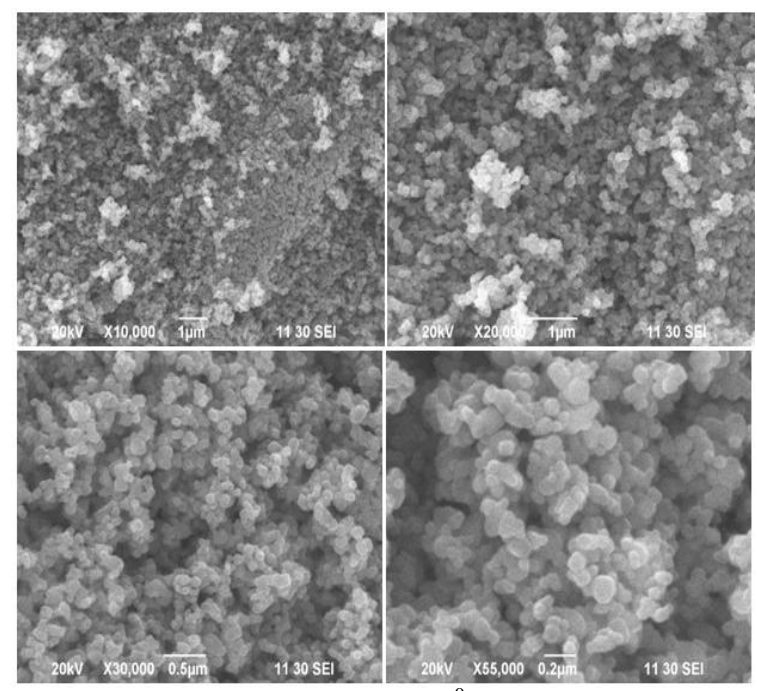

Fig 28: SEM of $1: 2,400^{\circ} \mathrm{C} \mathrm{S}-\mathrm{TiO}_{2} \mathrm{pc}$
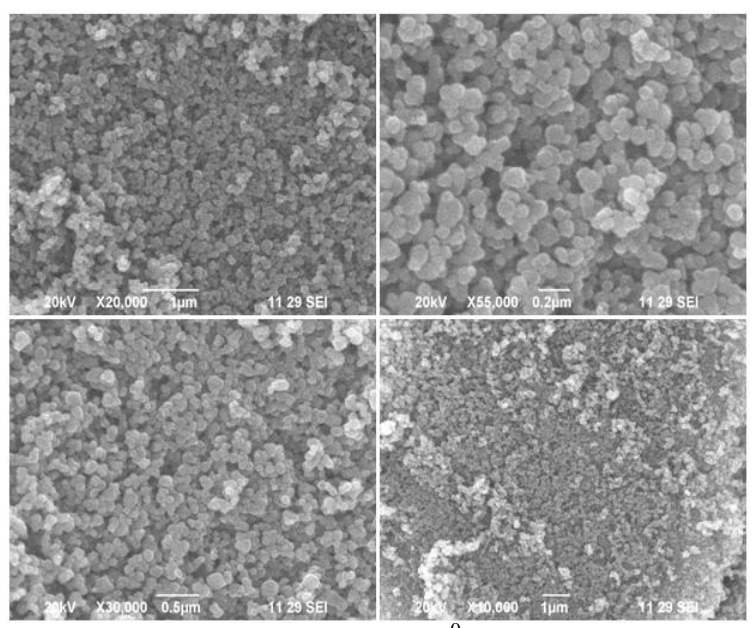

Fig 29: SEM of $1: 1,500^{\circ} \mathrm{C} \mathrm{S}-\mathrm{TiO}_{2} \mathrm{pc}$

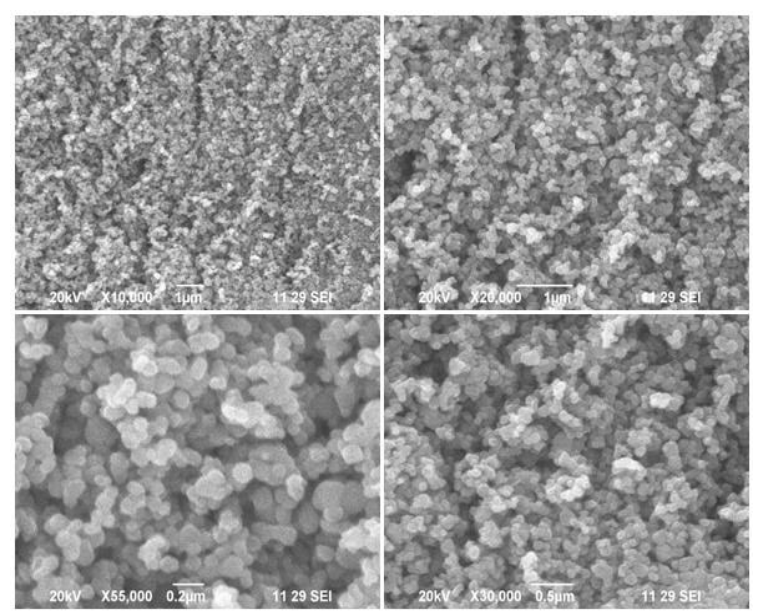

Fig 30: $\mathrm{SEM}$ of $1: 2,500^{\circ} \mathrm{C} \mathrm{S}-\mathrm{TiO}_{2} \mathrm{pc}$

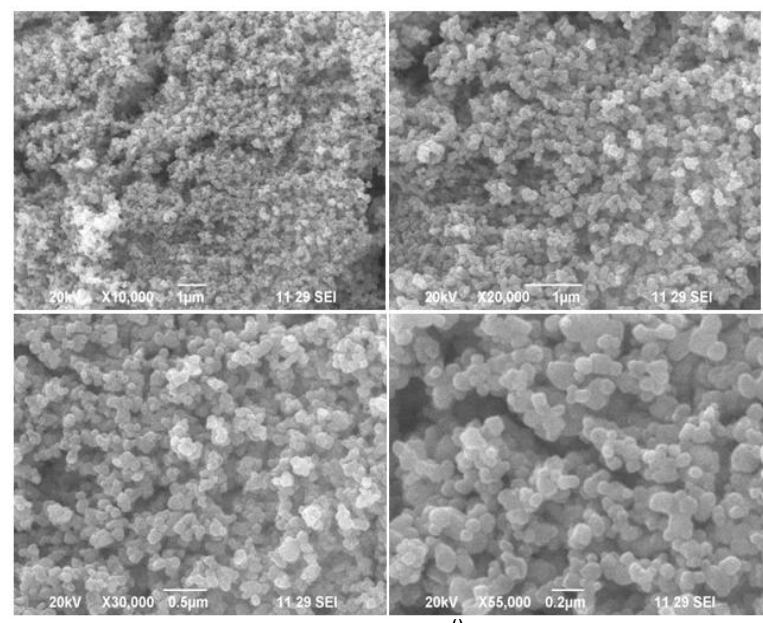

Fig 31: SEM of $1: 1,600^{\circ} \mathrm{C} \mathrm{S}-\mathrm{TiO}_{2} \mathrm{pc}$ 


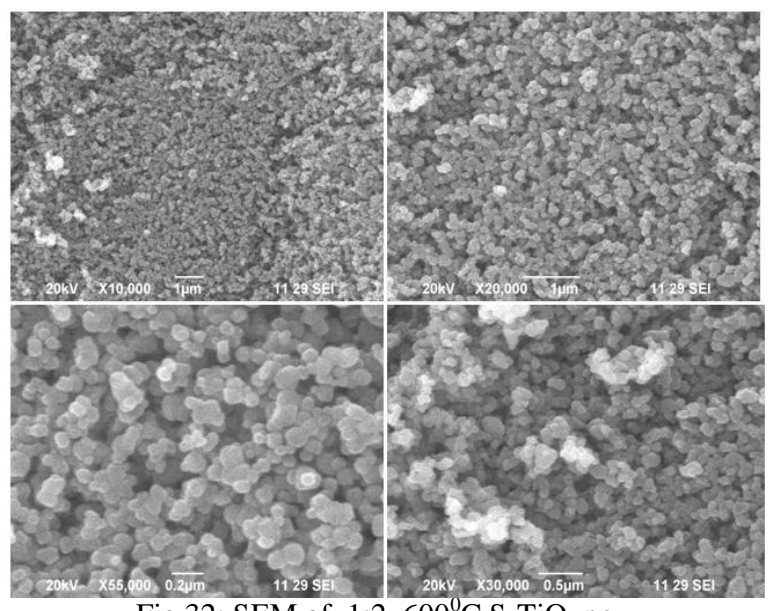

Fig 32: SEM of $1: 2,600^{\circ} \mathrm{C} \mathrm{S}-\mathrm{TiO}_{2} \mathrm{pc}$

Infrared spectroscopy of sulphur doped titanium dioxide pc

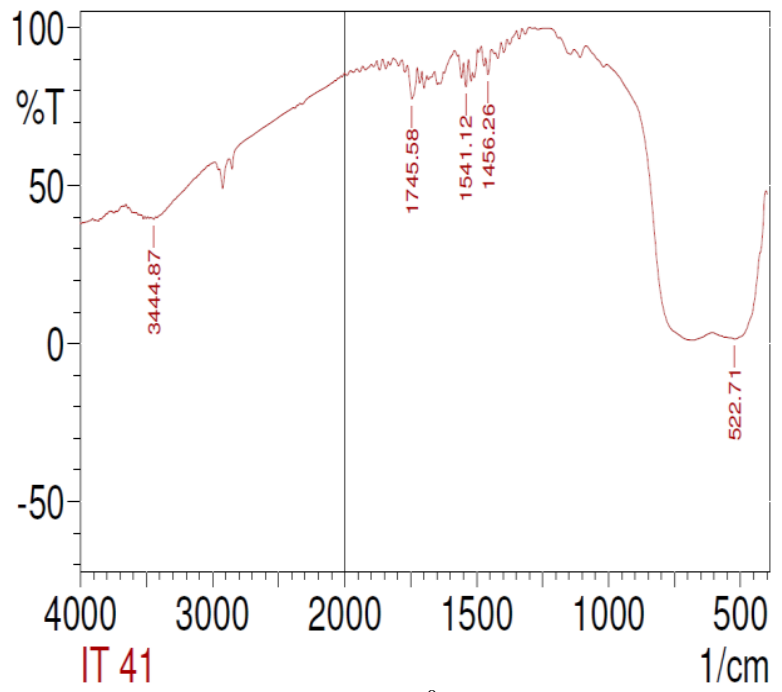

Fig33: IR of $1: 1,400^{\circ} \mathrm{C} \mathrm{S}-\mathrm{TiO}_{2} \mathrm{pc}$

\begin{tabular}{|l|l|l|}
\hline Peak & Intensity & $\begin{array}{l}\text { Correction } \\
\text { intensity }\end{array}$ \\
\hline 522.71 & 1.436 & 0.568 \\
\hline 1456.26 & 85.075 & 6.356 \\
\hline 1541.12 & 81.249 & 8.038 \\
\hline 3444.87 & 39.282 & 0.602 \\
\hline
\end{tabular}

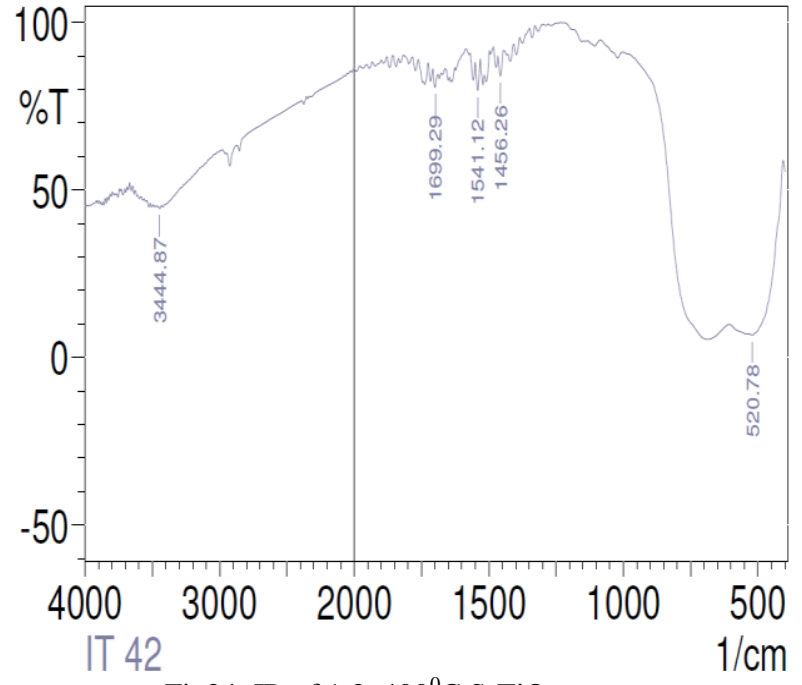

Fig34: IR of $1: 2,400^{\circ} \mathrm{C} \mathrm{S}-\mathrm{TiO}_{2}$ pc

\begin{tabular}{|l|l|l|}
\hline Peak & Intensity & $\begin{array}{l}\text { Correction } \\
\text { intensity }\end{array}$ \\
\hline 520.78 & 6.688 & 7.135 \\
\hline 1456.26 & 84.083 & 6.744 \\
\hline 1541.12 & 79.769 & 8.815 \\
\hline 1699.29 & 80.717 & 4.721 \\
\hline 3444.87 & 44.532 & 0.724 \\
\hline
\end{tabular}

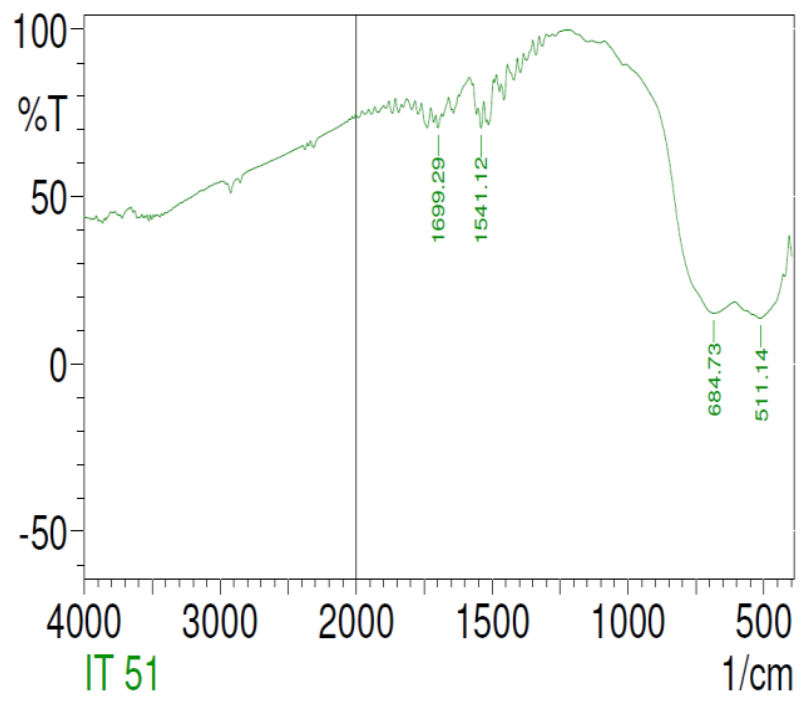

Fig35: IR of $1: 1,500^{\circ} \mathrm{C} \mathrm{S}-\mathrm{TiO}_{2} \mathrm{pc}$

\begin{tabular}{|l|l|l|}
\hline Peak & Intensity & $\begin{array}{l}\text { Correction } \\
\text { intensity }\end{array}$ \\
\hline 511.14 & 13.67 & 0.332 \\
\hline 684.73 & 15.176 & 17.098 \\
\hline 1541.12 & 70.502 & 6.916 \\
\hline 1699.29 & 70.557 & 4.03 \\
\hline
\end{tabular}




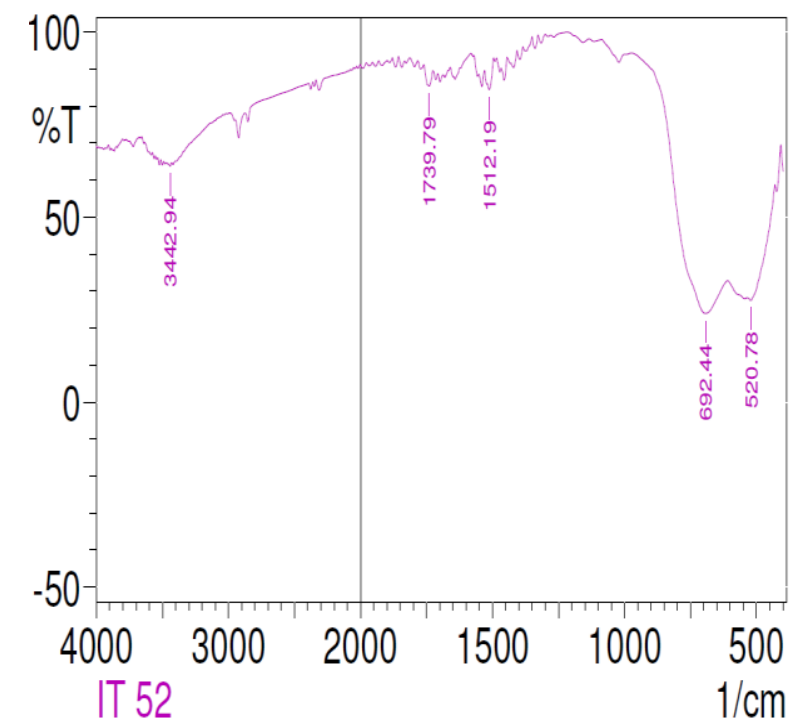

Fig36: IR of $1: 2,500^{\circ} \mathrm{C} \mathrm{S}-\mathrm{TiO}_{2} \mathrm{pc}$

\begin{tabular}{|l|l|l|}
\hline Peak & Intensity & $\begin{array}{l}\text { Correction } \\
\text { intensity }\end{array}$ \\
\hline 520.78 & 27.438 & 5.194 \\
\hline 692.44 & 23.934 & 22.922 \\
\hline 1512.19 & 84.364 & 3.371 \\
\hline 1739.79 & 85.339 & 4.834 \\
\hline 3442.94 & 63.799 & 0.736 \\
\hline
\end{tabular}

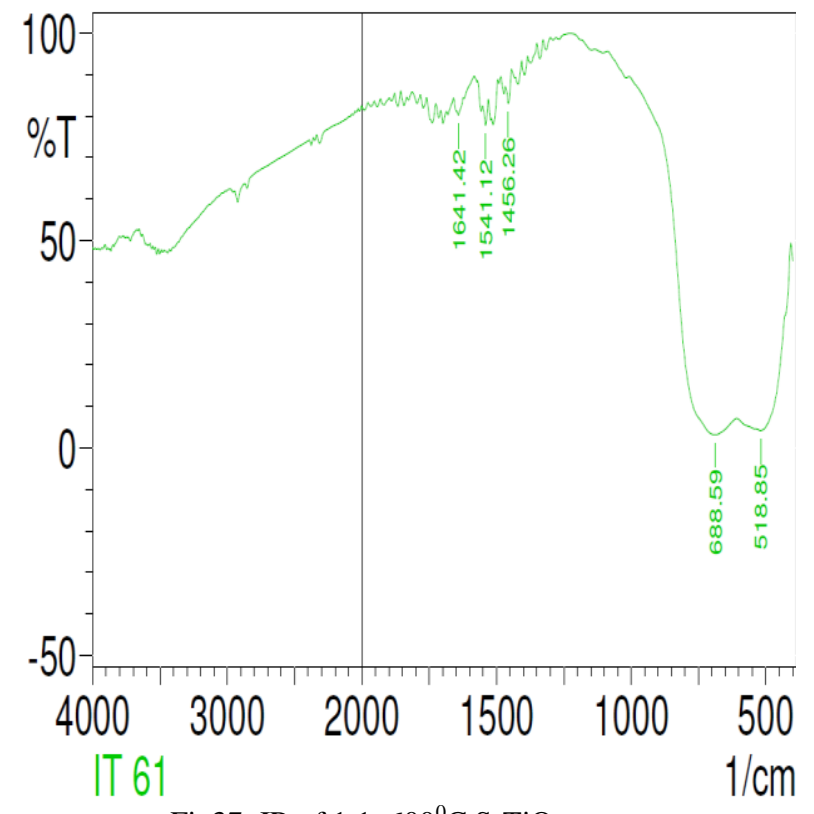

Fig37: IR of $1: 1,600^{\circ} \mathrm{C} \mathrm{S}-\mathrm{TiO}_{2} \mathrm{pc}$

\begin{tabular}{|l|l|l|}
\hline Peak & Intensity & $\begin{array}{l}\text { Correction } \\
\text { intensity }\end{array}$ \\
\hline 518.85 & 4.208 & 7.033 \\
\hline 688.59 & 3.191 & 20.659 \\
\hline 1456.26 & 83.056 & 6.186 \\
\hline 1541.12 & 77.793 & 5.375 \\
\hline 1641.42 & 80.217 & 1.807 \\
\hline
\end{tabular}

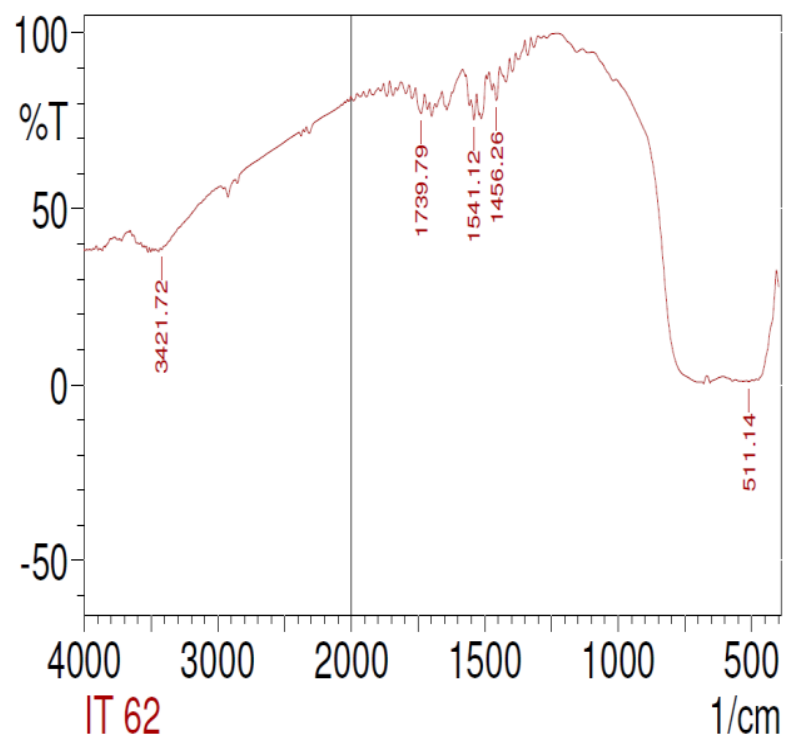

Fig38: IR of $1: 2,600^{\circ} \mathrm{C} \mathrm{S}-\mathrm{TiO}_{2} \mathrm{pc}$

\begin{tabular}{|l|l|l|}
\hline Peak & Intensity & $\begin{array}{l}\text { Correction } \\
\text { intensity }\end{array}$ \\
\hline 511.14 & 0.903 & 0.282 \\
\hline 1456.26 & 80.804 & 7.259 \\
\hline 1541.12 & 75.33 & 6.417 \\
\hline 1739.79 & 77.134 & 6.223 \\
\hline 3421.72 & 38.426 & 0.566 \\
\hline
\end{tabular}

X-Ray Diffraction Studies of sulphur doped titanium dioxide pc

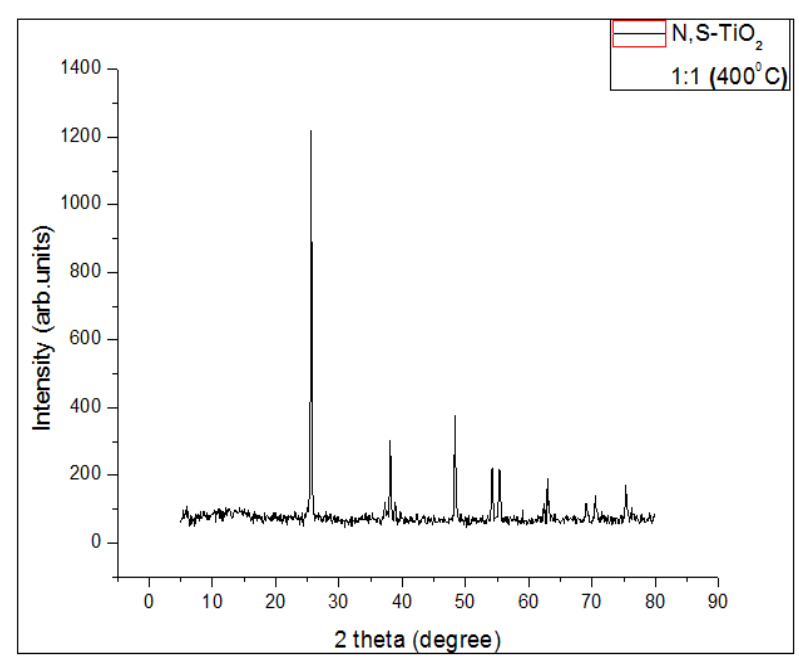

Fig.39 XRD of $1: 1,400^{\circ} \mathrm{C} \mathrm{S}-\mathrm{TiO}_{2} \mathrm{pc}$

\begin{tabular}{|l|l|l|}
\hline Strongest peak & 2theta(degree) & Intensity counts \\
\hline 1 & 25.5831 & 475 \\
\hline
\end{tabular}




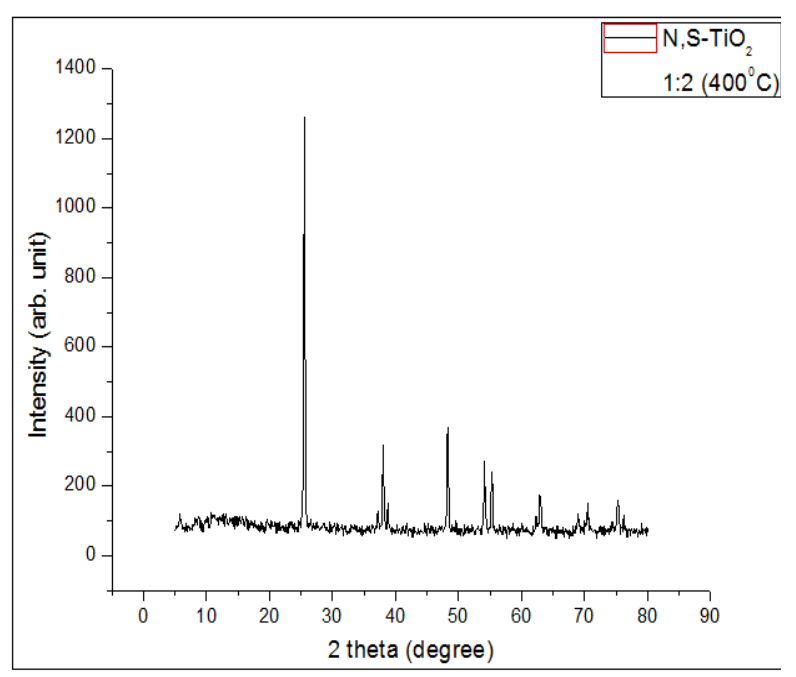

Fig.40 XRD of $1: 2,400^{\circ} \mathrm{C} \mathrm{S}-\mathrm{TiO}_{2} \mathrm{pc}$

\begin{tabular}{|l|l|l|}
\hline Strongest peak & 2theta(degree) & Intensity counts \\
\hline 1 & 25.5081 & 533 \\
\hline
\end{tabular}

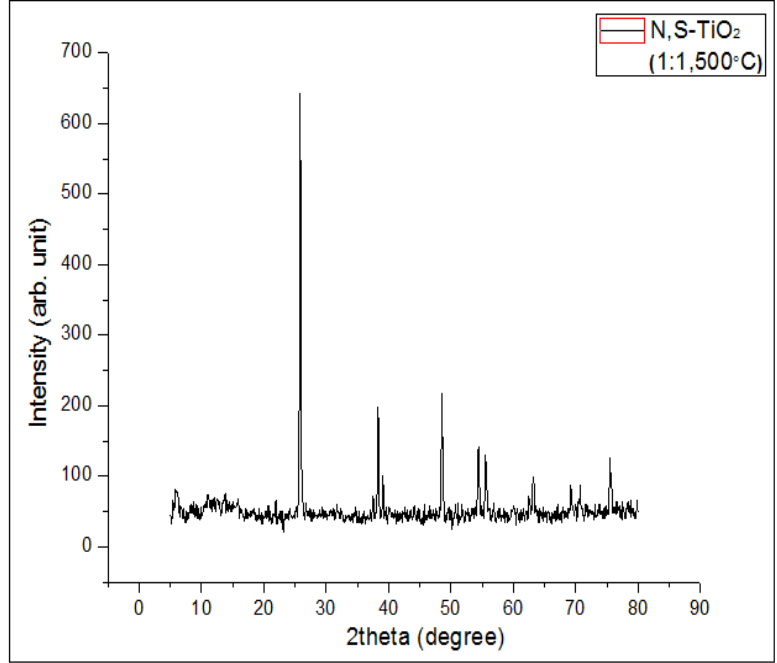

Fig.41 XRD of $1: 1,500^{\circ} \mathrm{C} \mathrm{S}-\mathrm{TiO}_{2} \mathrm{pc}$

\begin{tabular}{|l|l|l|}
\hline Strongest peak & 2 theta(degree) & Intensity counts \\
\hline 1 & 25.8070 & 270 \\
\hline
\end{tabular}

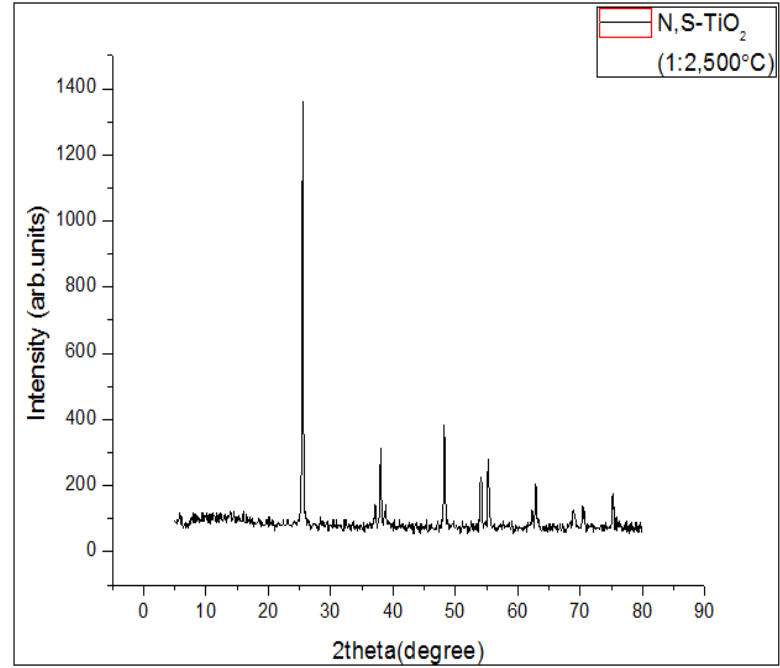

Fig. 42 XRD of $1: 2,500^{\circ} \mathrm{C} \mathrm{S}-\mathrm{TiO}_{2} \mathrm{pc}$

\begin{tabular}{|l|l|l|}
\hline Strongest peak & 2 theta(degree) & Intensity counts \\
\hline 1 & 25.4662 & 530 \\
\hline
\end{tabular}

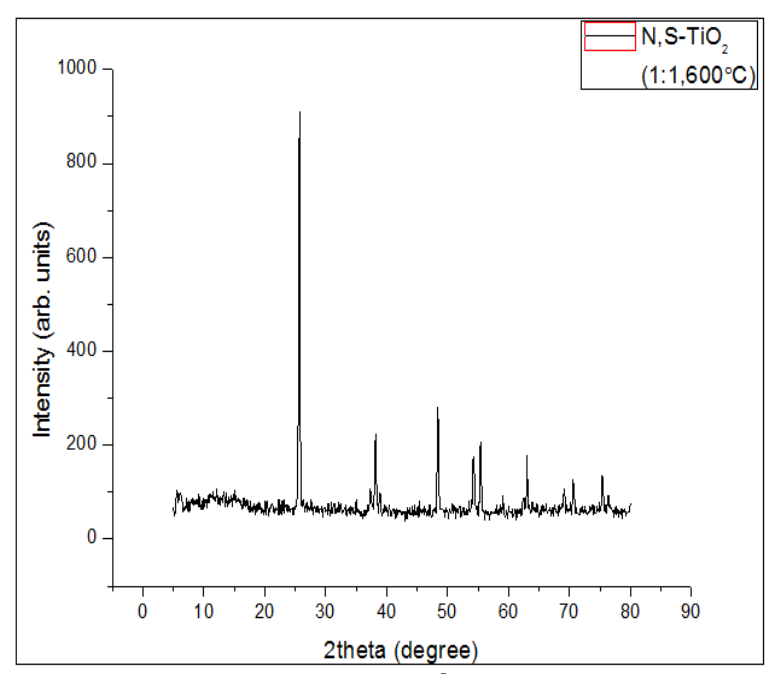

Fig.43 XRD of $1: 1,600^{\circ} \mathrm{C} \mathrm{S}-\mathrm{TiO}_{2} \mathrm{pc}$

\begin{tabular}{|l|l|l|}
\hline Strongest peak & 2theta(degree) & Intensity counts \\
\hline 1 & 25.6348 & 375 \\
\hline
\end{tabular}




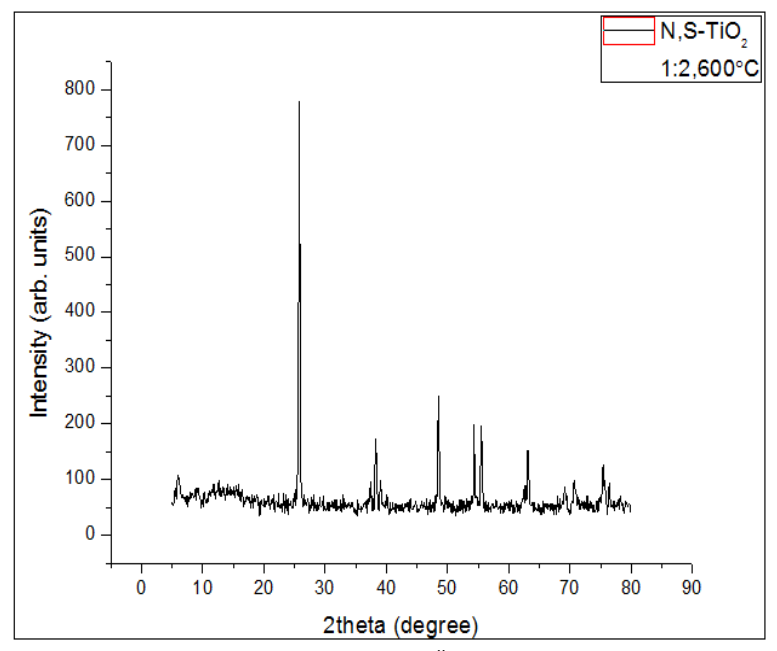

Fig.44 XRD of $1: 2,600^{\circ} \mathrm{C} \mathrm{S}-\mathrm{TiO}_{2} \mathrm{pc}$

\begin{tabular}{|l|l|l|}
\hline Strongest peak & 2 theta(degree) & Intensity counts \\
\hline 1 & 25.7501 & 309 \\
\hline
\end{tabular}

Table 1, Fig 1 reveals that the photodegradation of basic fuchsine dye in the dark shows the same value 0.956 for 3 hours. Table 2, Fig 2 reveals that the dye has photodegraded from 0.956 to 0.800 in light. Table 3, Fig 3 reveals that the dye has photodegraded from 0.956 to 0.368 in light with $0.01 \mathrm{~g}, 1: 1,400^{0} \mathrm{C} \mathrm{S}$ $\mathrm{TiO}_{2}$ pc. Table 4 , Fig 4 reveals that the dye has photodegraded from 0.956 to 0.47 in light with $0.01 \mathrm{~g}, 1: 2,400^{0} \mathrm{C} \mathrm{S}^{-} \mathrm{TiO}_{2}$ pc. Table 5, Fig 5 reveals that the dye has photodegraded from 0.956 to 0.269 in light with $0.01 \mathrm{~g}, 1: 1,500^{\circ} \mathrm{C} \mathrm{S}-\mathrm{TiO}_{2}$ pc. Table 6, Fig 6 reveals that the dye has photodegraded from 0.956 to 0.317 in light with $0.01 \mathrm{~g}, 1: 2,500^{0} \mathrm{C} \mathrm{S}-\mathrm{TiO}_{2} \mathrm{pc}$. Table 7, Fig7 reveals that the dye has photodegraded from 0.956 to 0.436 in light with $0.01 \mathrm{~g}, 1: 1,600^{\circ} \mathrm{C} \mathrm{S}$ $\mathrm{TiO}_{2}$ pc. Table 8 , Fig 8 reveals that the dye has photodegraded from 0.956 to 0.438 in light with $0.01 \mathrm{~g}, 1: 2,600^{\circ} \mathrm{C} \mathrm{S}-\mathrm{TiO}_{2}$ pc. Table 9, Fig 9 reveals that the dye has photodegraded from 0.956 to 0.404 in light with $0.02 \mathrm{~g}, 1: 1,400^{\circ} \mathrm{C} \mathrm{S}-\mathrm{TiO}_{2}$ pc. Table 10 , Fig 10 reveals that the dye has photodegraded from 0.956 to 0.428 in light with $0.02 \mathrm{~g}, 1: 2,400^{\circ} \mathrm{C} \mathrm{S}-\mathrm{TiO}_{2}$ pc. Table 11, Fig 11 reveals that the dye has photodegraded from 0.956 to 0.355 in light with $0.02 \mathrm{~g}, 1: 1,500^{0} \mathrm{C} \mathrm{S}-\mathrm{TiO}_{2}$ pc. Table 12 , Fig 12 reveals that the dye has photodegraded from 0.956 to 0.459 in light with $0.02 \mathrm{~g}, 1: 2,500^{\circ} \mathrm{C} \mathrm{S}-\mathrm{TiO}_{2}$ pc. Table 13 , Fig 13 reveals that the dye has photodegraded from 0.956 to 0.602 in light with $0.02 \mathrm{~g}, 1: 1,600^{\circ} \mathrm{C} \mathrm{S}-\mathrm{TiO}_{2}$ pc. Table 14, Fig 14 reveals that the dye has photodegraded from 0.956 to 0.629 in light with $0.02 \mathrm{~g}, 1: 2,600^{0} \mathrm{C} \mathrm{S}-\mathrm{TiO}_{2}$ pc. Table 15 , Fig 15 reveals that the dye has photodegraded from 0.956 to 0.717 in light with $0.03 \mathrm{~g}, 1: 1,400^{\circ} \mathrm{C} \mathrm{S}-\mathrm{TiO}_{2}$ pc. Table 16 , Fig 16 reveals that the dye has photodegraded from 0.956 to 0.796 in light with $0.03 \mathrm{~g}, 1: 2,400^{0} \mathrm{C} \mathrm{S}-\mathrm{TiO}_{2}$ pc. Table 17, Fig 17 reveals that the dye has photodegraded from 0.956 to 0.599 in light with $0.03 \mathrm{~g}, 1: 1,500^{\circ} \mathrm{C} \mathrm{S}-\mathrm{TiO}_{2}$ pc. Table 18 , Fig 18 reveals that the dye has photodegraded from 0.956 to 0.707 in light with $0.03 \mathrm{~g}, 1: 2,500^{\circ} \mathrm{C} \mathrm{S}-\mathrm{TiO}_{2}$ pc. Table 19 , Fig 19 reveals that the dye has photodegraded from 0.956 to 0.507 in light with $0.03 \mathrm{~g}, 1: 1,600^{\circ} \mathrm{C} \mathrm{S}-\mathrm{TiO}_{2}$ pc. Table 20, Fig 20 reveals that the dye has photodegraded from 0.956 to 0.546 in light with $0.03 \mathrm{~g}, 1: 2,600^{0} \mathrm{C} \mathrm{S}-\mathrm{TiO}_{2} \mathrm{pc}$.

\section{Conclusions}

Titanium dioxide has gained importance in recent researches for being an effective photocatalyst in many industries. Changing the dopants and experimenting for various research purposes can be of good scope now and in future.

\section{References}

[1] Thota and Tirukkovalluri, Visible Light Induced Photocatalytic Degradation of Methyl Red with Codoped Titania, Journal of Catalysts, 962419, 1-7, (2014).

[2] Kormann and Bahnemann, Preparation and characterization of quantum-size titanium dioxide, The Journal of Physical Chemistry, 92, 5196-5201, (1988)

[3] Lee and Kim, Anion co-doped Titania for Solar Photocatalytic Degradation of Dyes, Carbon Letters, Vol 9 (Issue 2), 131-136, (2008).

[4] Reddy and Baruwati, S-, N- and C-doped titanium dioxide nanoparticles: Synthesis, characterization and redox charge transfer study, Journal of Solid State Chemistry, 178, 3352-3358, (2005).

[5] Selvaraj and Parimiladevi, Synthesis of Nitrogen Doped Titanium Dioxide (TiO2) and its Photocatalytic Performance for the Degradation of Indigo Carmine Dye, Journal of Environmental and Nanotechnology, Vol 2 (Issue 1): 35-41, (2013). 